\title{
Novel microthermal sensor principle for determining the mixture ratio of binary fluid mixtures using Föppl vortices
}

\author{
B. Schmitt ${ }^{1}$, C. Kiefer ${ }^{1, *}$, and A. Schütze ${ }^{1}$ \\ ${ }^{1}$ Laboratory for Measurement Technology, Saarbrücken, Germany \\ * now at: Chair of Micromechanics, Microfluidics/Microactuators, Saarbrücken, Germany
}

Correspondence to: B. Schmitt (b.schmitt@lmt.uni-saarland.de)

Received: 10 March 2015 - Revised: 18 May 2015 - Accepted: 20 May 2015 - Published: 18 June 2015

\begin{abstract}
A novel sensor principle for determining binary fluid mixtures of known components is presented, making use of different thermal and rheological properties of the mixture's components. Using a microheater, a heat pulse is introduced in the mixture. The resulting temperature increase depends on the thermal properties of the mixture, allowing determination of the mixture ratio. Placing a bluff body in the fluid channel causes the formation of a stationary pair of vortices behind the body. The length of the vortex pair depends on the mixture's viscosity and thus its composition. By placing the microheater in the vortex area and making use of forced convection which changes with the size of the vortex, the sensitivity for determination of the mixture ratio can be increased by a factor of 2.5 compared to the direct thermal measurement. The flow velocity is measured independently of the mixture ratio using time-of-flight thermal anemometry.
\end{abstract}

\section{Introduction}

In many technical systems, monitoring and control of fluid mixtures is important for proper operation, while the cost of the sensor has to be very low. One potential application is the control of the mixture ratio of methanol in water for Direct Methanol Fuel Cells (DMFC), a technology that is promising to replace common batteries used in mobile applications (Dyer, 2002). Ren et al. (2000) and Dyer (2002) found out that the optimum concentration of methanol to be supplied to the fuel cell is approx. $1 \mathrm{M}$, corresponding to a volume fraction of approx. $4.2 \%$, to achieve optimum efficiency and long lifetime of the cell. This fraction is reduced as methanol is consumed in the cell. The optimal fraction then needs to be restored by adding pure methanol from a storage tank to achieve maximum energy density. Thus, Ren et al. (2000) stated that a sensor is required to measure the remaining methanol fraction and to determine the amount of methanol to be added.

Another potential application can be found in the exhaust aftertreatment of diesel cars using selective catalytic reduction (SCR) to reduce $\mathrm{NO}_{x}$ emission. Here, a solution of
$32.5 \%(\mathrm{~W} / \mathrm{W})$ of urea in water is injected into the exhaust stream in front of the catalyst to react with the $\mathrm{NO}_{x}$ to harmless $\mathrm{N}_{2}$ and water (Trautwein, 2003). According to Trautwein (2003), this is the most promising way of reducing $\mathrm{NO}_{x}$ emission without decreasing the engine's efficiency. A suitable sensor could allow the identification of the correct mixture to alert in case of wrong fuelling or to prevent conscious deception.

In both applications, low cost and long lifetime are main requirements for suitable sensor systems. To ensure this, a simple physical sensor principle for determining the mixture ratio is investigated because of the inherently greater stability and higher robustness compared to chemical sensor principles. First, sensor designs developed and experiments performed by Schmitt et al. (2013a) which were based on direct measurement of thermal fluid properties, have shown promising results, but improved sensitivity and accuracy are required to meet the application requirements. The presented measurement principle is subject to patent application (Kiefer et al., 2015). 


\section{Measurement principle}

When a bluff body or baffle is placed in a fluid flow, different types of flow past the obstacle can occur, depending on the present Reynolds number Re (Föppl, 1913; Lienhard, 1966; Hucho, 2011). This is calculated with the (unimpaired) flow velocity $u_{0}$, the characteristic length $d$ of the bluff body and the kinematic viscosity $v$, see Eq. (1).

$R e=u_{0} \frac{d}{v(\varphi, T)}$

Here, the dependence of the viscosity on temperature $T$ and volume fraction $\phi$ of the two components in the mixture is considered. At very low Reynolds numbers close to zero, socalled creeping or unseparated flow past the obstacle is observed, which is characterized by a smooth flow without the presence of turbulence or vortices. With increasing Reynolds numbers, a stationary and symmetric pair of vortices (also called Föppl vortices) develops, forming a wake behind the obstacle, as illustrated in Fig. 1. The resulting total flow velocity along the symmetry axis is also shown schematically. Since the fluid flows in the opposite direction in the wake, a point $x_{T}$ exists at which the flow is zero. This point denotes the length of the vortex pair and it is used in this work for determining the fluid mixture ratio. Based on the work of Van Dyke (1988), Hucho (2011) showed that in the case of circular cylinders, the length of the vortex pair depends linearly on $R e$ for $R e$ lower than approx. 50. Similar behaviour has been reported for equilateral triangular cylinders by Shademani et al. (2013) based on finite volume simulations, while Zielinska and Wesfreid (1995) determined that for these obstacles, the critical Reynolds number is approx. 38. For higher Reynolds numbers the well-known Kármán vortex street is formed, with its typical repeating pattern of swirling vortices that are caused by the unsteady separation of fluid flow. This effect is used by vortex flow sensors to measure the flow velocity, since the frequency $f$ of the vortex shedding is given by

$f=S t \frac{u_{0}}{d}$,

with the Strouhal number St (Webster,1999). At even higher Reynolds numbers the fluid flow becomes turbulent.

The novel measurement principle investigated here makes use of the changing length of the stationary vortex pair, which depends on the Reynolds number and hence also on flow velocity $u_{0}$ and kinematic viscosity $v(\phi, T)$. If flow velocity and temperature are known, the viscosity and thus the mixture ratio of two known components in a mixture can be determined based on the length of the vortex pair. Considering methanol/water mixtures, the vortex principle promises a higher sensitivity vs. the methanol fraction than other measurement principles, as the viscosity shows a much higher influence of the mixture ratio compared to the relative permittivity or thermal properties such as the thermal conductivity (cf. Table 1). The only physical property with higher

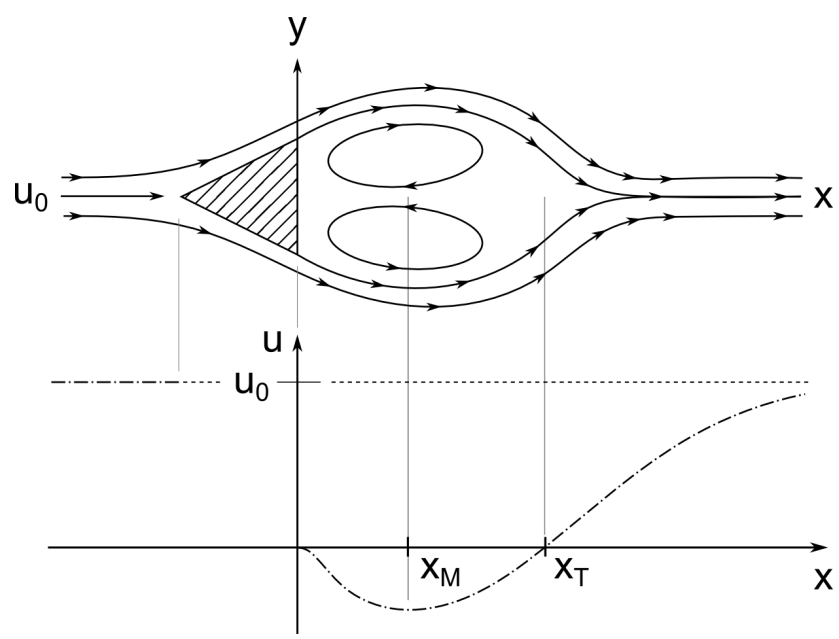

Figure 1. Schematic representation of fluid flow past a triangular bluff body at medium Reynolds numbers, causing Föppl vortices and the resulting total flow velocity along the symmetry axis.

sensitivity is the electrical conductivity $\sigma$, which, however, is also highly sensitive to surface contamination and therefore considered less robust.

The determination of the vortex pair length is based on the effect of forced convection which depends on the local flow velocity above a heated area. Here, a resistive microheater is placed at $x_{T}$ and excited, e.g. using a constant current, resulting in an increase in temperature. Since the local flow velocity at the end of the vortex pair is close to zero, heat is mainly dissipated by conduction, which is influenced by the thermal properties like thermal conductivity and heat capacity, as shown by Schmitt and Schütze (2014). If the length of the vortex pair changes due to a change in viscosity and/or flow velocity, the magnitude of the local flow velocity over the heater increases. Thus, additional heat is dissipated due to forced convection and the resulting heater temperature decreases. By using multiple microheaters located as an array along the symmetry axis behind the bluff body, as shown in Fig. 2, the heater showing the highest temperature increase indicates the length of the vortices. If the flow velocity is known, knowledge of the vortex length allows the determination of the mixture ratio. To address applications with varying flow velocity this can be determined simultaneously by a time-of-flight method investigated by Schmitt et al. (2013a) in an earlier work. Here, the temperature sensor (depicted in green on the right side at the end of the heater array in Fig. 2) is designated to be used to detect the temperature pulse induced by one of the heaters of the array. The time required for the pulse to reach the temperature sensor is inversely proportional to the flow velocity and unaffected by the mixture ratio which influences the thermal properties of the fluid, as shown by Schmitt et al. (2013a). The second temperature sensor (depicted in green on the left side in front of the bluff body; $\mathrm{cf}$. Fig. 2) is used to determine the reference temperature of the 
Table 1. Relative sensitivity vs. changing methanol fraction of some physical properties for methanol/water mixtures (at $25^{\circ} \mathrm{C}$ ).

\begin{tabular}{lrl}
\hline Property & $\begin{array}{r}\text { Change in } \% \\
\text { per } \%(\mathrm{~W} / \mathrm{W})\end{array}$ & Ref. \\
\hline Electrical conductivity $\sigma$ & $+5.1 \ldots 10.9^{\mathrm{a}}$ & Thomas and Mandeville (1937) \\
Kinematic viscosity $\nu^{\mathrm{b}}$ & +3.1 & Mikhail and Kimel (1961) \\
Thermal conductivity $\lambda$ & -0.87 & Bates et al. (1938) \\
Relative permittivity $\varepsilon$ & -0.55 & Albright and Gosting (1946) \\
Density $\rho$ & -0.17 & Thomas and Mandeville (1937) \\
\hline
\end{tabular}

a The cited reference does not specify the exact concentration notation. ${ }^{\mathrm{b}}$ Calculated from dynamic viscosity and density.

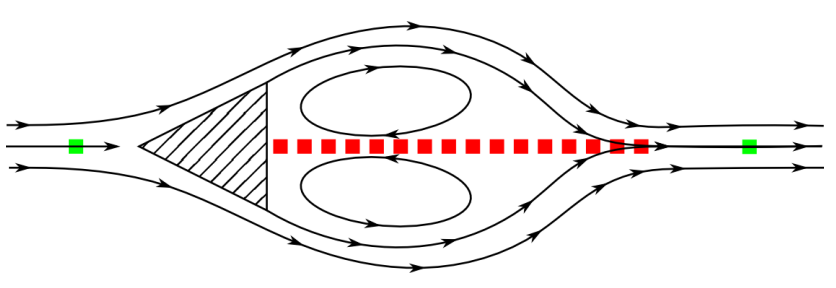

Figure 2. Schematic presentation of the array of microheaters (depicted in red) and two additional temperature sensors (green).

fluid. This is especially required since the viscosity of water shows a very high sensitivity to temperature of approx. $-2 \% \mathrm{~K}^{-1}$, according to Mikhail and Kimel (1961).

\section{Experimental setup}

\subsection{Sensor layout}

The sensor layout used and an enlarged view of the active area are shown in Figs. 3 and 4, respectively. The meandershaped resistive sensing elements are made of sputtered aluminium on a polyimide foil with a thickness of $125 \mu \mathrm{m}$. The array in the middle can be heated in individual areas with a size of $0.4 \times 0.4 \mathrm{~mm}^{2}$ each which are overlapping the neighbouring heaters by $50 \%$, resulting in an array of 16 heaters with a total length of $3.4 \mathrm{~mm}$. Each individual heater is excited with a constant current while its temperature increase is measured. As depicted in Fig. 4, the red wires are used to excite the heater while the rest of the meanders and the green contact wires are used to determine its exact resistance with four-terminal sensing and with its temperature. The temperature sensor placed in front of the bluff body is used to measure the reference temperature of the fluid. To realize the aforementioned time-of-flight method to determine the flow velocity, the last heater of the array, where the fluid flow is again undisturbed, and the temperature sensor at the end of the chip are used. Note that the two additional temperature sensors also have four contact wires each, allowing four-terminal sensing for exact temperature measurements. The accuracy achieved for the temperature measurement of the heaters and temperature sensors is approx. $0.1 \mathrm{~K}$.

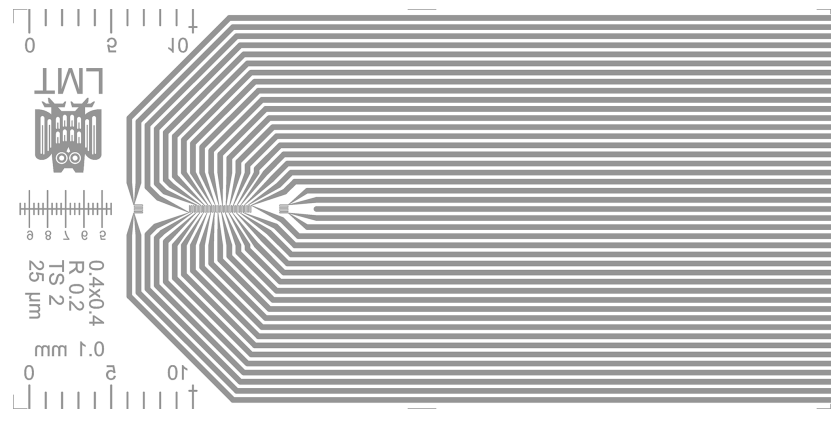

Figure 3. Developed sensor layout with heater array and two distinct temperature sensors. The chips are contacted using FPC connectors (Schmitt et al., 2013b).

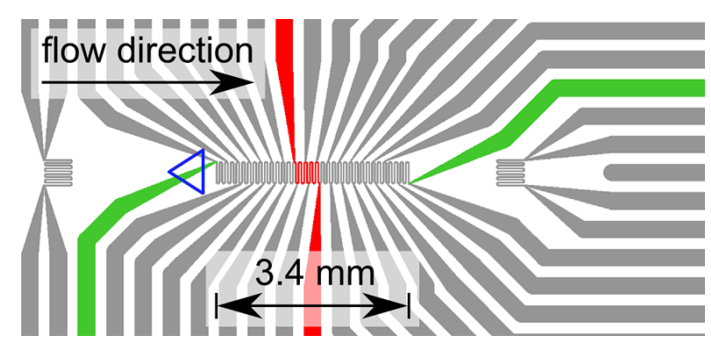

Figure 4. Enlarged view of the developed sensor layout with triangular bluff body (Schmitt et al., 2013b).

The thickness of the aluminium structures is approx. $0.5 \mu \mathrm{m}$. In addition, a $40 \mathrm{~nm}$ thick titanium layer was used to improve the adhesion of the polyimide foil. The structures are passivated by spin-coating with a polyamide-imide resin (Durimide 32A, Fujifilm). A photograph of a realized sensor chip is shown in Fig. 5. The resistance of a single heater area is approx. $12 \Omega$ at room temperature; the temperature coefficient was determined to be $3.25 \times 10^{-3} \mathrm{~K}^{-1}$ for a reference temperature of $0^{\circ} \mathrm{C}$. 

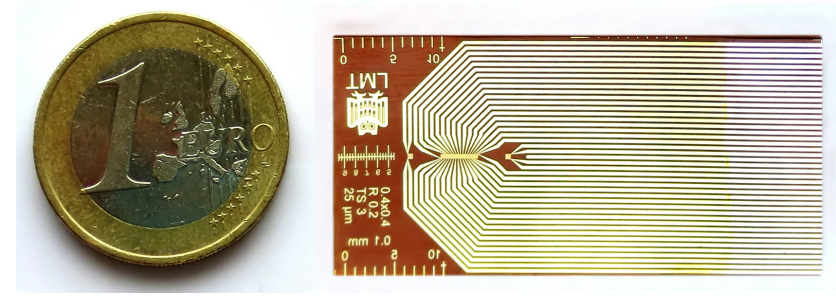

Figure 5. Photograph of sensor chip realized on polyimide foil (Schmitt et al., 2013b).

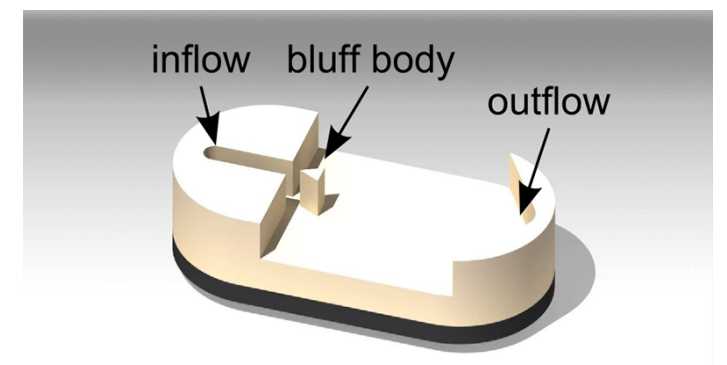

Figure 6. Insert manufactured using 3-D printing with integrated triangular bluff body and sealing on the bottom side (Schmitt et al., 2013b).

\subsection{Measurement setup}

To generate the stationary vortices, the insert with the integrated triangular bluff body, shown in Fig. 6, was fabricated using 3-D printing technology. This part is inserted into the cavity of the measurement chamber, shown in Fig. 7. The insert has a length of $17.5 \mathrm{~mm}$ and a width of $8.5 \mathrm{~mm}$. The inflow is realized by a channel with a cross section of $1 \times 1 \mathrm{~mm}^{2}$ to increase the flow velocity in front of the bluff body, and hence increase the Reynolds number, in order to have a longer vortex pair. The triangular bluff body used has a base length and height of $1 \mathrm{~mm}$ each and is placed with a gap of $0.5 \mathrm{~mm}$ between its tip and the end of the inflow channel. The bottom side of the insert is covered with a softer material to act as a sealing. The bore holes which allow in- and outflow at the beginning of the inflow channel and at the end of the insert through its base are hidden in this view. Syringe pumps (C3000, TriContinent) were used to feed the system with the methanol/water mixtures to achieve very low pulsation and exact control of the flow rate. The control of the system and data acquisition using a USB-6009 data acquisition system (National Instruments) was implemented in LabVIEW (National Instruments).

\subsection{Measuring process}

The investigated mixtures of methanol in deionized water with volume fractions of 0,5 and $10 \%$ were prepared 1 day

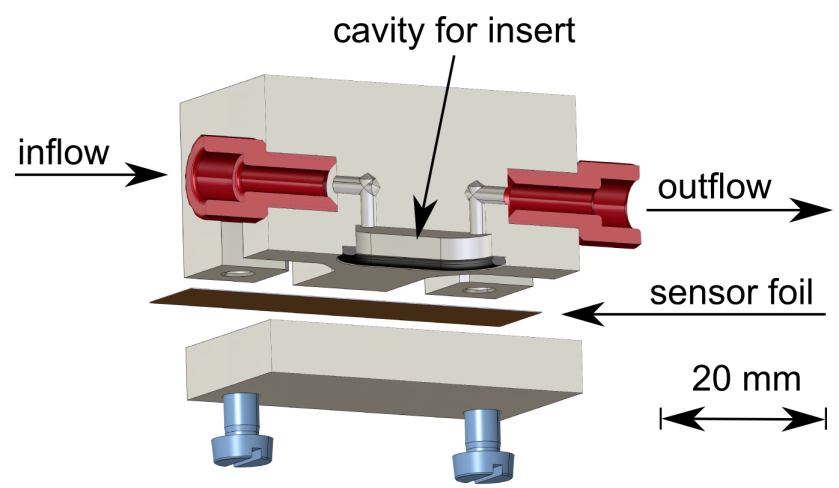

Figure 7. 3-D cross-sectional view of the measurement chamber used (according to Schmitt et al., 2013b).

before the measurements due to the mixing enthalpy, which causes a temperature increase when mixing methanol and water. The sensor substrate was positioned underneath the insert at the bottom of the measurement chamber, resulting in a small gap of approx. $0.1 \mathrm{~mm}$ between the first heater of the array and the back side of the bluff body. During the measurements at an ambient (reference) temperature of $28^{\circ} \mathrm{C}$, the applied flow rate was kept constant at values between 0.01 and $0.1 \mathrm{~mL} \mathrm{~s}^{-1}$. Due to the cross section of the insert inflow channel of $1 \times 1 \mathrm{~mm}^{2}$, these flow rates correspond to a mean flow velocity in this channel between 0.01 and $0.1 \mathrm{~m} \mathrm{~s}^{-1}$. The temperatures of the two separate temperature sensors were measured by applying a constant current of $5 \mathrm{~mA}$ corresponding to approx. $0.4 \mathrm{~mW}$. Using this low power, no temperature increase was observed after applying the current, even without fluid flow. For the heaters in the array, a heating current of approx. $65 \mathrm{~mA}$ was applied for $3 \mathrm{~s}$ to each heater of the array successively, beginning with the heater with the largest distance from the bluff body. This corresponds to an initial heating power of approx. $50 \mathrm{~mW}$, which increased to approx. $58 \mathrm{~mW}$ during heating due to the increase of the heater resistance with temperature. To take the slight differences of the individual heater resistances $R_{n}$ of the $n$ elements into account, the current $I_{n}$ of each element was adapted so that the same heating power $P=R_{n} \times I_{n}^{2}$ is applied at room temperature. Note that the heating power increases during the heat pulse due to the increase of the heater resistance, but the resulting heating power curve is the same again for all heater elements. After changing the flow rate, a break of $5 \mathrm{~s}$ was applied to allow the vortex pair to fully develop before starting the next measurement. Also, the first heater was preheated for $5 \mathrm{~s}$ to compensate for the fact that the following ones are affected by the previous heaters due to their overlapping. Finally, the heater temperature was averaged over the last $2 \mathrm{~s}$ of heating for every individual heater. Since every individual heater is heated for $3 \mathrm{~s}$, the measurement time for a full sweep of all 16 individual heaters re- 


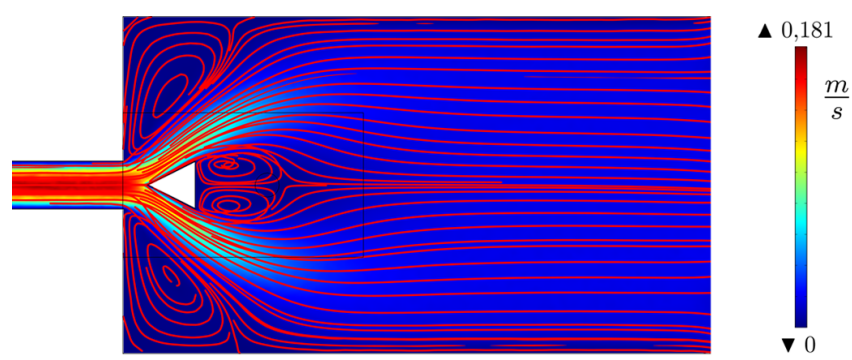

Figure 8. Simulated fluid flow past the triangular bluff body for water with a flow rate of $0.07 \mathrm{~mL} \mathrm{~s}^{-1}$, with colours indicating velocity magnitude and streamlines depicted in red.

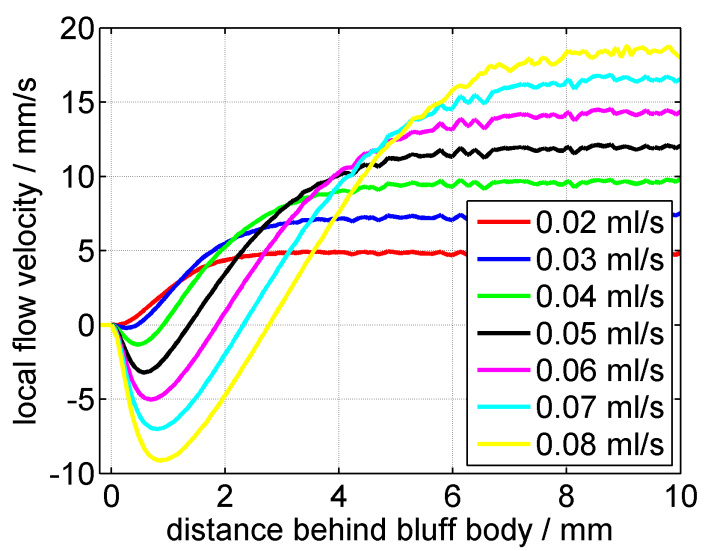

Figure 9. Simulation of local flow velocity behind the triangular bluff body along the symmetry axis for different flow rates of water.

quires $50 \mathrm{~s}$, which is too long for many applications. However, this can be shortened, as discussed in the conclusion.

\section{Simulations}

Finite element simulations of the measurement principle were performed in Comsol Multiphysics (Comsol AB) using the conjugate heat transfer interface. Figure 8 shows the simulated velocity magnitude and streamlines for water at a flow rate of $0.07 \mathrm{~mL} \mathrm{~s}^{-1}$ at an ambient temperature of $25^{\circ} \mathrm{C}$. As expected, a pair of stationary vortices forms behind the bluff body. Figures 9 and 10 show the simulated local flow velocity along the symmetry axis behind the triangular bluff body for different flow rates of water and for different methanol fractions at a constant flow rate of $0.08 \mathrm{~mL} \mathrm{~s}^{-1}$, respectively. From Fig. 9 we can deduce that a flow rate of at least $0.04 \mathrm{~mL} \mathrm{~s}^{-1}$ is required to cause the formation of a vortex pair as evidenced by the negative flow velocity behind the bluff body. As expected from the analytical model, in the simulated flow rate and fraction ranges the simulations show a nearly linear dependence of the length of the vortex pair on the flow rate and an inversely proportional dependence on the methanol fraction (which causes a nearly linear change in viscosity), respectively, as shown in Fig. 11. In Fig. 12 the

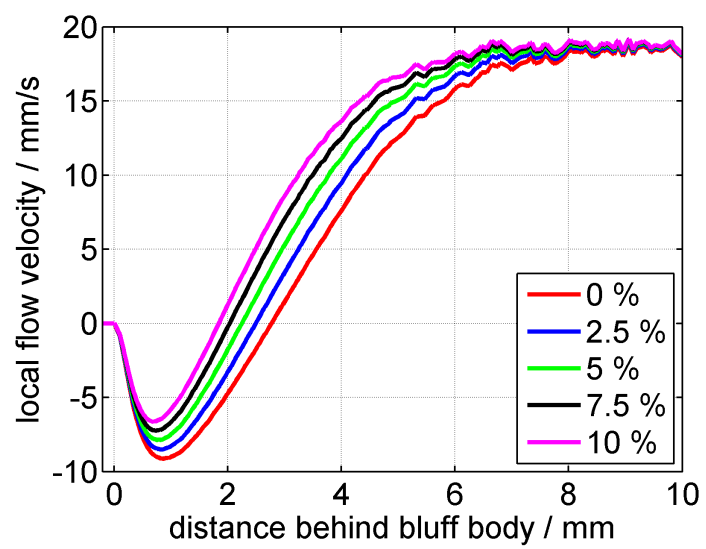

Figure 10. Simulation of the local flow velocity behind the triangular bluff body along the symmetry axis for different fractions of methanol in water at a flow rate of $0.08 \mathrm{~mL} \mathrm{~s}^{-1}$.

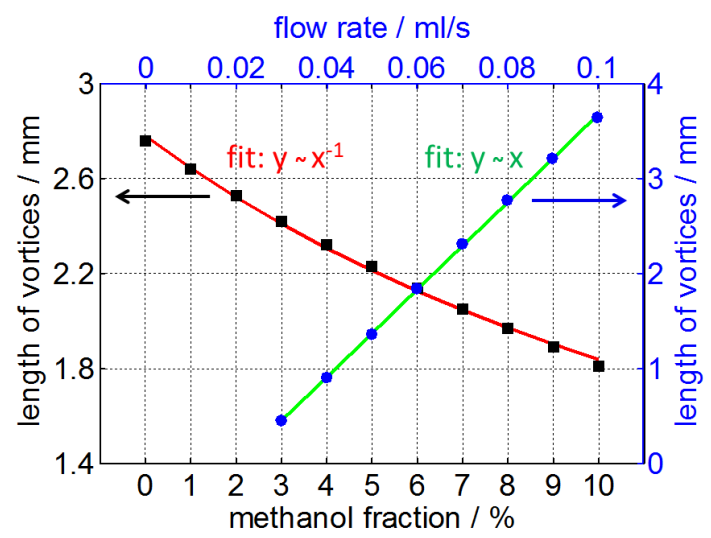

Figure 11. Simulated dependence of the stationary vortex pair length on flow rate of water and methanol fraction at a flow rate of $0.08 \mathrm{~mL} \mathrm{~s}^{-1}$.

simulated temperature increase of individual heaters with diameters of $0.5 \mathrm{~mm}$, heated with a constant power of $30 \mathrm{~mW}$, is shown for different positions behind the bluff body for a flow rate of $0.08 \mathrm{~mL} \mathrm{~s}^{-1}$ for two volume fractions of 5 and $10 \%$ methanol in water. The different shape of the heaters to the realized layout was used due to former simulations without using stationary vortices. As expected, the resulting local heater temperatures show a maximum which is located at the point where the local flow velocity is zero, cf. Fig. 10. It has to be noted here that the heaters were not simulated as an array of successively heated areas, but as single heaters. Thus, the influence of a former heated area on the temperature increase of other heaters is not considered here. Furthermore, the heat dissipation into the substrate underneath the heaters is neglected; thereby the simulated temperatures of the heaters are only qualitatively comparable with the measurements.

The simulations show that placing a heater inside the wake decreases the length of the vortices by less than about $3 \%$. 


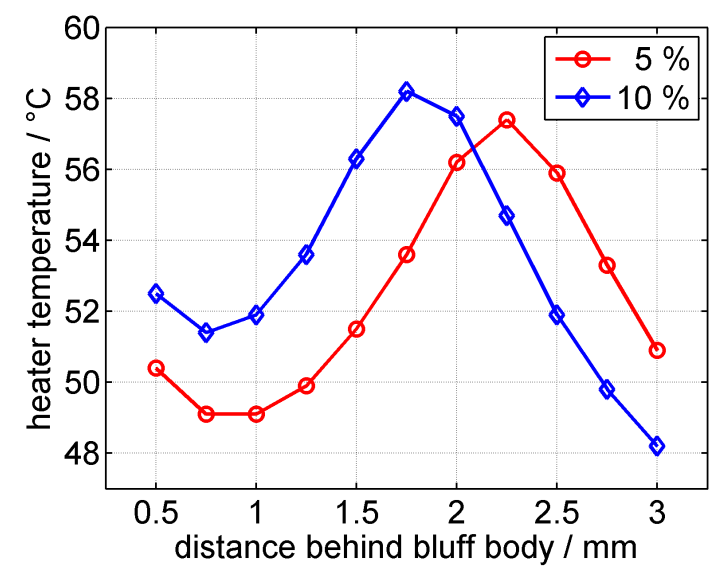

Figure 12. Simulated local temperature increase of individual heaters located at different positions behind the bluff body for 5 and $10 \%$ methanol in water, respectively, for a constant flow rate of $0.08 \mathrm{~mL} \mathrm{~s}^{-1}$.

On the other hand, the local flow velocity inside the wake is strongly affected and shows an increase of up to $20 \%$, depending on the apparent flow rate. The penetration depth of the temperature field into the fluid is less than $0.5 \mathrm{~mm}$.

\section{Measurement results and discussion}

In Fig. 13 the measured temperature increase of the individual heaters of the array as a function of their distance from the bluff body is shown for different flow rates of water. At a flow rate of $0.01 \mathrm{~mL} \mathrm{~s}^{-1}$, no influence of the vortex pair is evident. At this low flow rate, the apparent Reynolds number calculated from Eq. (1) with the base length of the wedge of $1 \mathrm{~mm}$, the cross section of the channel in which the vortices are formed of $8.5 \mathrm{~mm}^{2}$ and the kinematic viscosity at $25^{\circ} \mathrm{C}$ of $0.894 \times 10^{-6} \mathrm{~m}^{2} \mathrm{~s}^{-1}$, is 1.3 and results in creeping flow, while no vortex pair is formed. The slightly higher temperature of the farthest heater $3.3 \mathrm{~mm}$ behind the bluff body is caused by a longer heating time used for this heater, which is heated first. At a flow rate of $0.04 \mathrm{~mL} \mathrm{~s}^{-1}$ a small influence of the vortices can be seen and the apparent heater temperatures are lower due to the increased cooling by forced convection. For higher flow rates the temperature curves show distinct maxima, indicating the length of the vortex pair where the local flow velocity is nearly zero. As expected, the length of the vortex pair increases with increasing flow rate. The higher temperatures observed close to the bluff body can be explained by a shadowing effect of the bluff body and a resulting lower flow velocity close to zero that is also shown in the simulations (cf. Fig. 9).

Figure 14 shows the measured temperature increase for three volume fractions of methanol in water at constant flow rates of 0.01 and $0.09 \mathrm{~mL} \mathrm{~s}^{-1}$. At the lowest flow rate, the larger temperature increase observed for higher fractions of methanol is due to the reduced thermal effusivity of the mix-

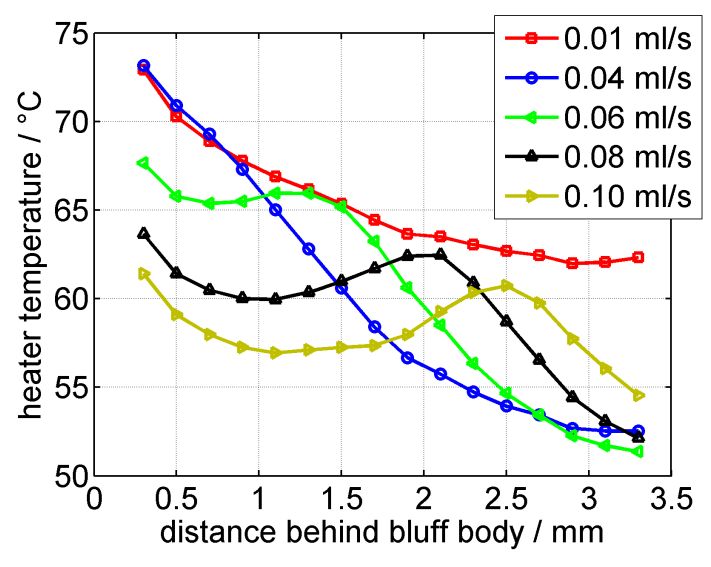

Figure 13. Measured temperature increase (averaged over the last $2 \mathrm{~s}$ of every heat pulse) of the individual heaters of the array for different flow rates of water.

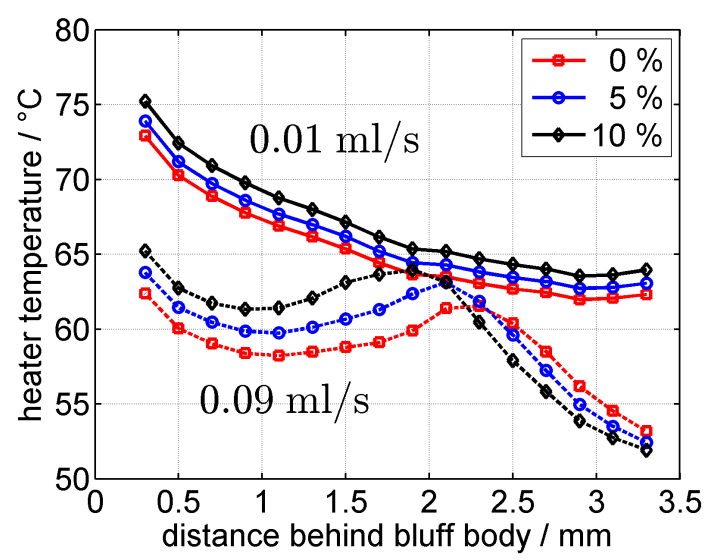

Figure 14. Measured temperature increase (averaged over the last $2 \mathrm{~s}$ of every heat pulse) of the individual heaters of the array for different volume fractions of methanol in water for constant flow rates of 0.01 and $0.09 \mathrm{~mL} \mathrm{~s}^{-1}$.

ture. At a flow rate of $0.09 \mathrm{~mL} \mathrm{~s}^{-1}$ the influence of the vortices is visible. With increasing methanol fraction the viscosity of the mixture also increases, resulting in a shortening of the vortex pair, as evidenced by the measurement data.

Figure 15 shows the measured sensitivity of the heater temperatures vs. the methanol fraction for flow rates of 0.06 and $0.09 \mathrm{~mL} \mathrm{~s}^{-1}$. Evidently, the position showing the highest sensitivity shifts to the right with increasing flow rate and also depends on the concentration range. In Fig. 16 the sensitivity calculated for a change from 0 to $5 \%$ of methanol in water is shown for different flow rates between 0.01 and $0.10 \mathrm{~mL} \mathrm{~s}^{-1}$. At a very low flow rate, the sensitivity is nearly independent of the position and corresponds to the sensitivity for undisturbed flow of approx. $0.2 \mathrm{~K}^{-1}$, which is comparable to earlier results using similar microheaters in a stopped fluid (Schmitt et al., 2013a). With increasing flow rate, the sensitivity initially decreases for all heaters. When 


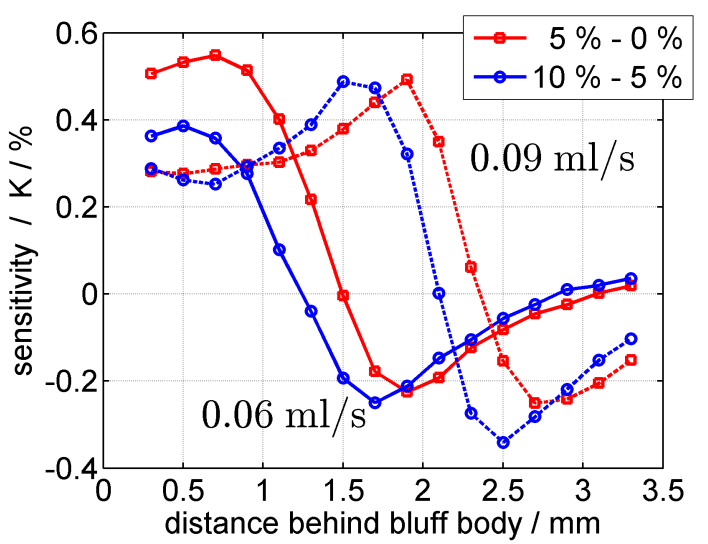

Figure 15. Measured sensitivity (defined as change in heater temperature divided by change in methanol fraction) of the individual heaters of the array for two different flow rates of 0.06 and $0.09 \mathrm{~mL} \mathrm{~s}^{-1}$. Two sensitivity curves are shown for a change in the methanol fraction from 0 to $5 \%$ and from 5 to $10 \%$, respectively.

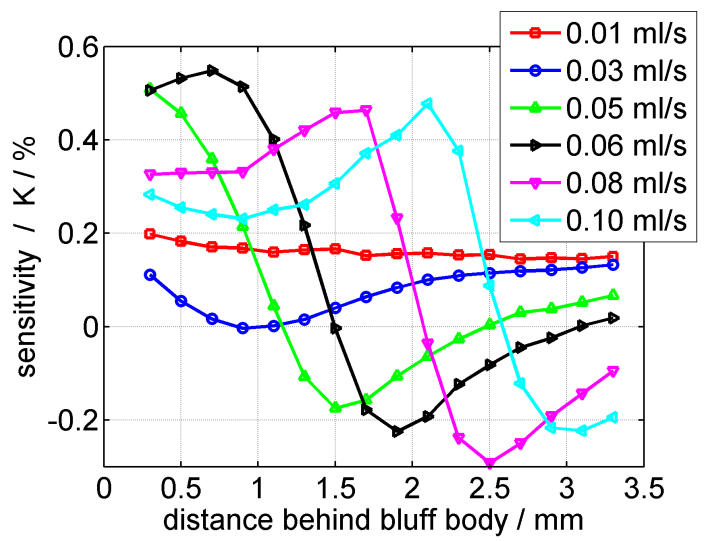

Figure 16. Measured sensitivity (defined as change in heater temperature divided by change in methanol fraction) of the individual heaters of the array for different flow rates.

the vortex pair is formed at flow rates of $0.05 \mathrm{~mL} \mathrm{~min}^{-1}$ and higher, the maximum sensitivity is much higher compared to undisturbed flow. The required number of heaters can be reduced in order to obtain a simpler layout without a significant loss of sensitivity. With only four heaters at distances of $0.5,1.0,1.5$ and $2.0 \mathrm{~mm}$ behind the bluff body and for flow rates between 0.5 and $1.0 \mathrm{~mL} \mathrm{~min}^{-1}$, a sensitivity of at least $0.04 \mathrm{~K}^{-1}$ is achieved, i.e. twice as high as without the vortex pair.

In Fig. 17 the measured length of the vortex pair is shown, based on the position of the heater in the array showing the temperature maximum. Here, no interpolation between two heaters was used, which could improve the determination of the vortex pair length. In addition, a linear fit of the curve representing $5 \%$ of methanol in water is shown. The expected linear correlation between the vortex length and the flow rate

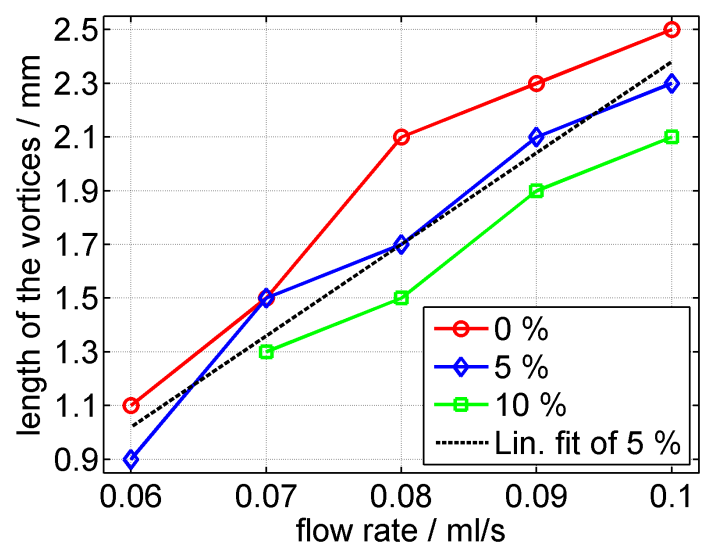

Figure 17. Length of the stationary vortex pair for different flow rates evaluated for three volume fractions of methanol in water.

is evident with deviations from the ideal linear dependence that are less than the spatial resolution of the heaters.

When interpolating the curves shown in Fig. 14, the length of the vortex pair decreases from approx. 2.22 to $1.90 \mathrm{~mm}$ when the methanol fraction increases from 0 to $10 \%$, resulting in a sensitivity of $32 \mu \mathrm{m} \%^{-1}$. However, when regarding the measurement data, it is evident that due to the flat top of the local maximum of the curves, exact determination of the vortex pair length is very difficult. Regarding the influence of the flow rate on the length of the vortices for water and assuming a linear correlation, an increase from $1.19 \mathrm{~mm}$ at $0.06 \mathrm{~mL} \mathrm{~s}^{-1}$ to $2.47 \mathrm{~mm}$ at $0.1 \mathrm{~mL} \mathrm{~s}^{-1}$ is observed, resulting in a sensitivity of $32 \mathrm{~mm}\left(\mathrm{~mL} \mathrm{~s}^{-1}\right)^{-1}$. In a previous work, Schmitt et al. (2013a) showed that using the proposed time-of-flight measurement principle for determination of the flow rate achieved a measurement uncertainty of less than $1.34 \mu \mathrm{L} \mathrm{s}^{-1}$ in the flow rate range between 0.027 and $0.055 \mathrm{~mL} \mathrm{~s}^{-1}$ which is relevant for DMFCs. Using error propagation, this results in a measurement uncertainty of the volume fraction of approx. $1.34 \%$ due to the uncertainty of the flow rate. The measurement uncertainty for determining the mixture ratio neglecting the influence of the fluid flow is expected to be in the range of $0.5 \%$, as has been achieved in previous experiments without the use of a bluff body (Schmitt et al., 2013a). In addition, the viscosity of the fluid is strongly influenced by temperature, resulting in a decrease of approx. $2 \% \mathrm{~K}^{-1}$ at room temperature. Thus, the accuracy achieved for the temperature measurement of the heaters and temperature sensors of approx. $0.1 \mathrm{~K}$ results in an additional measurement uncertainty of the viscosity and hence the mixture ratio of $0.2 \%$. In total, the expected overall uncertainty is approx. $2 \%$ if both flow rate and reference temperature have to be determined simultaneously.

It is evident that the required measurement time for the serial heating of the heater array presented here is far too long for most applications. An alternative and more feasible approach is to first determine the apparent flow rate us- 
ing the time-of-flight principle, followed by the evaluation of the temperature increase of the heater in the array that has the highest sensitivity to the methanol fraction for the determined flow rate. Considering a flow rate of $0.09 \mathrm{~mL} \mathrm{~s}^{-1}$ as presented in Fig. 14, the heater at a distance of $1.7 \mathrm{~mm}$ behind the bluff body shows the highest sensitivity vs. a change in the methanol fraction of approx. $0.5 \mathrm{~K} \%^{-1}$. This represents an increase in sensitivity by a factor of 2.5 compared to measurements based on thermal measurements alone, without using vortices as reported by Schmitt et al. (2013a).

Comparing the simulations and experiments, the measured length of the vortex pair is approx. $30 \%$ shorter than was simulated. The observed reduced length is attributed to the simulated simplified geometry, such as the triangular bluff body used in the experimental setup having rounded edges due to the fabrication process using a 3-D printer. Considering the rounded edges in the simulations, the length of the vortex pair is reduced by about $10 \%$ at a flow rate of $0.07 \mathrm{~mL} \mathrm{~s}^{-1}$. With increasing flow rate, the influence of the rounding decreases.

First preliminary evaluations of the reproducibility of the measurements resulted in uncertainties of $\pm 0.25 \mathrm{~K}$. In combination with the sensitivity of $0.5 \mathrm{~K}^{-1}$ this results in a measurement accuracy of $0.5 \%$ for the methanol volume fraction.

\section{Conclusion}

A novel measurement principle to determine the mixture ratio of binary fluid mixtures based on stationary vortices forming a wake behind a bluff body has been presented. In finite element simulations, the correlation between the length of the vortex pair and the Reynolds number predicted by analytical models has been confirmed. Experiments have verified the simulated results at least qualitatively. Quantitative differences can be attributed to different reference temperatures, the imperfect geometry of the 3-D printed bluff body and the influence of neighbouring heaters on each other, but further investigations are needed. Evaluating the length of the vortex pair using the proposed heater array directly is difficult because a very high spatial resolution is needed. Since no mathematical model has yet been developed to describe the local temperature distribution behind the bluff body, no physically inspired fit of the measured temperature distribution is possible to increase the resolution. In addition, this would require the measurement of multiple heaters, which requires a longer measurement time. Alternatively, when the flow rate is known, the heater providing the highest sensitivity to the mixture ratio can be evaluated, thus reducing the required measurement time to $3 \mathrm{~s}$, or even less. When doing so, the resolution is evaluated to be $0.5 \%$ if flow rate and reference temperature are known. If both have to be determined additionally, a lower resolution of approx. $2 \%$ is achieved. Furthermore, the sensor can be simplified significantly by re- ducing the number of heaters without a significant loss of sensitivity.

Assuming a critical Reynolds number of 38 according to Zielinska and Wesfreid (1995), the maximum flow rate for the generation of Föppl vortices is approx. $0.29 \mathrm{~mL} \mathrm{~s}^{-1}$ at $25^{\circ} \mathrm{C}$. In this case the sensitivity vs. the methanol concentration would be greatly reduced if only one heater without vortices were used, as proposed by Schmitt et al. (2013a). Thus, the applicable flow rate range can be extended with the new concept based on Föppl vortices.

Due to the required vortex pair, the response time of the novel measurement principle after a change in the methanol fraction has to be evaluated in further experiments to determine the achievable measurement rate. Furthermore, vortices forming in the corners of the insert (cf. Fig. 8) could cause carry-over effects. Here, an improvement of the sensor chamber geometry is probably required. Furthermore, adhesion of air bubbles in the flow channel can cause disturbances of the fluid flow and has to be prevented. Finally, vibrations are expected to occur in the intended application of DMFCs as well as in automotive applications. Their influence on the measurement principle also has to be investigated in the future.

Acknowledgements. Funding of parts of this research by the German Federal Ministry of Education and Research (BMBF) in the framework of the project InMischung (support code: 16SV5394) in the Microsystems Technology program is gratefully acknowledged.

Edited by: N.-T. Nguyen

Reviewed by: two anonymous referees

\section{References}

Albright, P. S. and Gosting, L. J.: Dielectric Constants of the Methanol-Water System from 5 to $55^{\circ}$, J. Am. Chem. Soc., 68, 1061-1063, doi:10.1021/ja01210a043, 1946.

Bates, O. K., Hazzard, G., and Palmer, G.: Thermal Conductivity of Liquids, Ind. Eng. Chem. Anal. Ed., 10, 314-318, doi:10.1021/ac50122a006, 1938.

Dyer, C. K.: Fuel cells for portable applications, J. Power Sources, 106, 31-34, doi:10.1016/S0378-7753(01)01069-2, 2002.

Föppl, L.: Wirbelbewegung hinter einem Kreiszylinder, Verl. d. K. B. Akad. d. Wiss., München, 1913.

Hucho, W. H.: Aerodynamik der stumpfen Körper, 2. Auflage, Vieweg + Teubner, Springer, Wiesbaden, Germany, 2011.

Kiefer, C., Schmitt, B., and Schütze, A.: Verfahren und Vorrichtung zur Bestimmung der Viskosität einer in einem Strömungskanal strömenden Flüssigkeit, unexamined patent application, application number DE 102013019872 A1, 2015.

Lienhard, J. H.: Synopsis of Lift, Drag, and Vortex Frequency Data for Rigid Circular Cylinders, Bulletin 300, Technical Extension Service, Washington State University, USA, 1966.

Mikhail, S. Z. and Kimel, W. R.: Densities and Viscosities of Methanol-Water Mixtures, J. Chem. Eng. Data, 6, 533-537, doi:10.1021/je60011a015, 1961. 
Ren, X., Zelenay, P., Thomas, S., Davey, J., and Gottesfeld, S.: Recent advances in direct methanol fuel cells at Los Alamos national laboratory, J. Power Sources, 86, 111-116, doi:10.1016/S0378-7753(99)00407-3, 2000.

Schmitt, B. and Schütze, A.: Analytische Beschreibung von Hot Disk-Sensoren zur Bestimmung von Methanolkonzentration und Strömungsgeschwindigkeit für Direktmethanolbrennstoffzellen, Symposium des Arbeitskreises der Hochschullehrer für Messtechnik, Saarbrücken, Germany, 18-20 September 2014, 15-24, doi:10.5162/AHMT2014/1.2, 2014.

Schmitt, B., Kiefer, C., and Schütze, A.: Microthermal sensors for determining fluid composition and flow rate in fluidic systems, in: Proc. SPIE 8763, Grenoble, France, 17 May 2013, 87630O, doi:10.1117/12.2017293, 2013a.

Schmitt, B., Kiefer, C., and Schütze, A.: Neuartiger thermischer Wirbelsensor zur Bestimmung von binären Mischungsverhältnissen in Fluiden, 11. Dresdner Sensorsymposium, Dresden, Germany, 9-11 December 2013, 373-378, doi:10.5162/11dss2013/F16, 2013b.
Shademani, R., Ghadimi, P., Zamanian, R., and Dashtimanesh, A.: Assessment of Air Flow over an Equilateral Triangular Obstacle in a horizontal Channel Using FVM, J. Math. Sci. Appl., 1, 1216, doi:10.12691/jmsa-1-1-3, 2013.

Thomas M. K. and Mandeville, D. C.: The electrical conductivity of potassium chloride in methyl alcohol-water mixtures, P. Indian Acad. Sci. A, 6, 312-315, doi:10.1007/BF03051254, 1937.

Trautwein, W. P.: DGMK-Research Report 616-1, Deutsche Wissenschaftliche Gesellschaft für Erdöl, Erdgas und Kohle e.V., Hamburg, 2003.

Van Dyke, M.: An Album of Fluid Motion, Fourth Printing, The Parabolic Press, Stanford, California, USA, 1988.

Webster, J. G. (Ed.): The Measurement, Instrumentation, and Sensors Handbook, CRC Press LLC, Boca Ranton, Florida, USA, 1999.

Zielinska, B. J. A. and Wesfreid, J. E.: On the spatial structure of global modes in wake flow, Phys. Fluids, 7, 1418-1424, doi:10.1063/1.868529, 1995. 